\title{
Oxytocin in the paraventricular nucleus attenuates incision-induced mechanical allodynia
}

\author{
YANFENG ZHANG $^{1}$, YONG YANG ${ }^{1}$, RUPING DAI $^{2}$, HUI WU $^{3}$, CHANGQI LI $^{4}$ and QULIAN GUO ${ }^{1}$ \\ ${ }^{1}$ Department of Anesthesiology, Xiangya Hospital, Central South University, Changsha, Hunan 410078; \\ ${ }^{2}$ Department of Anesthesiology, The Second Xiangya Hospital, Central South University, Changsha, Hunan 410011; \\ ${ }^{3}$ Department of Medical Oncology, Hunan Provincial Tumor Hospital, The Affiliated Tumor Hospital, \\ Xiangya School of Medicine, Central South University, Changsha, Hunan 410013; \\ ${ }^{4}$ Department of Anatomy and Neurobiology, Xiangya School of Medicine, \\ Central South University, Changsha, Hunan 410078, P.R. China
}

Received May 9, 2014; Accepted October 21, 2014

DOI: $10.3892 /$ etm.2015.2285

\begin{abstract}
Oxytocin (OT) neurons localized in the paraventricular nucleus (PVN) and supraoptic nucleus (SON) send fibers to the brain and spinal cord. While most previous studies have looked at the role of OT in chronic pain, few have investigated the role of OT in acute pain, particularly postoperative pain. In the present study, the role of OT in incision-induced allodynia was explored for the first time, using a rat incisional pain model. Immunohistochemical staining showed that, compared with the baseline (prior to incision) measurements, the OT content in the PVN was significantly decreased at $0.5,1.0$ and $3.0 \mathrm{~h}$ post-incision and returned to the baseline level at $6.0 \mathrm{~h}$ post-incision. By contrast, there was no significant difference in the OT content in the SON prior to and subsequent to incision. A dose-dependent inhibition of mechanical hypersensitivity was detected $30 \mathrm{~min}$ after intracerebroventricular injection of OT (100, 400 or $600 \mathrm{ng}$ ) and lasted for $3.0 \mathrm{~h}$. No significant difference was noted, however, between the intrathecal OT injection group (600 ng) and the control group. In conclusion, the present study provides the first in vivo evidence that OT in the PVN predominantly attenuates incision-induced mechanical allodynia at the supraspinal, rather than the spinal, level. This suggests that OT is involved in supraspinal analgesia for postoperative pain.
\end{abstract}

Correspondence to: Dr Qulian Guo, Department of Anesthesiology, Xiangya Hospital, Central South University, 87 Xiangya Road, Changsha, Hunan 410008, P.R. China

E-mail: anesthesiacsu@gmail.com

Key words: oxytocin, incisional pain, mechanical allodynia, supraspinal analgesia, paraventricular nucleus, supraoptic nucleus, spinal cord

\section{Introduction}

The neuropeptide oxytocin (OT) is synthesized within the hypothalamus in the paraventricular nucleus (PVN) and supraoptic nucleus (SON) (1). Central OT plays an important role in modulating a variety of physiological functions in mammals, including parturition, lactation, social behavior and memory (2).

Accumulating evidence shows that OT has analgesic properties as a neurotransmitter or neuromodulator (3). Anatomically, OT neurons in the PVN send direct descending fibers to several brain regions involved in pain perception (4), including the dorsal and ventral hippocampus, amygdala, periaqueductal gray (PAG) and raphe nuclei, as well as the superficial dorsal horn of the spinal cord $(1,5)$. In addition, extensive clinical and preclinical studies have demonstrated that OT in the PVN, rather than that in the SON, is involved in anti-nociception or analgesia $(6,7)$. It has been reported that the intrathecal injection of OT can significantly reduce withdrawal responses to mechanical and cold stimulation in sciatic nerve-ligated rats, and the effect can be blocked by intrathecal injection of an OT antagonist $(6,7)$. In humans, Madrazo et al (8) reported that the intractable thoracic pain of a patient with diffuse mesothelioma could be reduced by $88 \%$ for $77 \mathrm{~min}$ by intracerebroventricular injection of OT. Yang (9) reported that acute and chronic lower back pain in humans resulted in a marked change of OT content in the cerebral spinal fluid and plasma; OT could relieve this lower back pain, and the effect of OT was blocked by an OT antagonist.

While most previous studies have looked at the role of OT in chronic pain, few investigations have been carried out into the role of oxytocin in acute pain, particularly postoperative pain. In the present study, the role of OT in incisional-induced allodynia was explored for the first time, to the best of our knowledge.

\section{Materials and methods}

Animals. The experiments were performed using male Sprague Dawley rats (200-250 g) provided by the Animal 
Experimental Center of Xiangya School of Medicine, Central South University (Changsha, China). The rats were housed singly with food and water available ad libitum in a temperature-controlled $\left(25 \pm 2^{\circ} \mathrm{C}\right)$ room and under a 12-h light/dark cycle. All experiments were conducted in accordance with the guidelines of the International Association for the Study of Pain (10). Every effort was made to minimize any suffering of the animals and the number of animals used.

Incisional pain model. The hindpaw incision model was performed as described by Brennan et al (11). The model and control groups each consisted of 30 mice. Briefly, rats were anesthetized with $2 \%$ isoflurane (Sigma-Aldrich, St. Louis, MO, USA) delivered through a nose cone. The plantar aspect of the right hindpaw was prepared in a sterile manner with a $10 \%$ povidone-iodine solution (Sigma-Aldrich), and the paw was positioned through a hole in a sterile drape. A $1-\mathrm{cm}$ longitudinal incision was made through the skin and fascia of the plantar aspect of the foot, with a size 11 blade, starting at a point $0.5 \mathrm{~cm}$ away from the proximal edge of the heel and extending toward the toes. The plantaris muscle was raised and incised longitudinally, with its origin and insertion remaining intact. The incision was closed with two mattress sutures of 5-0 nylon. Following surgery, the animals were allowed to recover in their home cages. Sham control groups, consisting of rats that received anesthesia, antiseptic preparation and topical antibiotic without an incision, were used in the study.

Intracerebroventricular injection of OT. Animals were anesthetized by intraperitoneal injection of chloral hydrate $(300 \mathrm{mg} / \mathrm{kg})$ and mounted on a stereotaxic frame. A stainless steel guide cannula $(0.8 \mathrm{~mm}$ outer diameter $)$ was directed into the lateral ventricle (anteroposterior, $-0.92 \mathrm{~mm}$; lateral, $1.3 \mathrm{~mm}$; dorsoventral, $4.0 \mathrm{~mm}$ ) according to the rat brain stereotaxic atlas of Paxinos and Watson (12). The cannula protruded $1 \mathrm{~cm}$ above the skull and was fixed to the skull by dental acrylic. Finally, the opening was sealed. If no abnormality was noted during the first three days after implantation, a stainless steel needle $(0.4 \mathrm{~mm}$ diameter) was inserted directly into the lateral ventricle along the guide cannula and $10 \mu \mathrm{l}$ vehicle $(0.9 \%$ saline) or OT $(100,400$ or $600 \mathrm{ng}$; Sigma-Aldrich) solution was injected over $10 \mathrm{~min}$ immediately after hindpaw incision.

Intrathecal injection of OT. Intrathecal catheter implantation was performed as previously described (13), with minor modifications. Following incision, OT (600 ng, total volume $10 \mu \mathrm{l}$ ) was injected intrathecally through the catheter, followed by a $10-\mu 1$ saline flush, and mechanical hypersensitivity was assessed.

Mechanical hypersensitivity assay. Von Frey filaments (Stoelting Co., Wood Dale, IL, USA) were used to assess the withdrawal threshold prior to incision and at $0.5,1.0,3.0,6.0$, 24.0 and $72.0 \mathrm{~h}$ after incision, according to the 'up-down' algorithm, as previously described (14). The assay was performed by an investigator blinded to the treatment. At the end of the experiments, $20 \mu 1$ methylthionine chloride was injected into the lateral ventricle via the cannula, and a coronal section was made across the lateral ventricle. Only data from rats whose ventricle system was filled with methylthionine chloride were included in the analysis.

Immunohistochemistry. Rats were deeply anesthetized with chloral hydrate $(300 \mathrm{mg} / \mathrm{kg})$ and perfused transcardially with $4 \%$ paraformaldehyde. The brain and lumbar enlargement from each rat were post-fixed with $4 \%$ paraformaldehyde for $4 \mathrm{~h}$, and then immersed overnight in $0.01 \mathrm{M}$ phosphate buffer containing 30\% sucrose. Cryostat-cut brain and lumbar spinal cord sections $(30-\mu \mathrm{m})$ were incubated overnight at room temperature with polyclonal rabbit anti-human anti-OT antibody (\#O4389; 1:2,000 dilution; Sigma-Aldrich). Subsequent to washing in $0.01 \mathrm{M}$ phosphate-buffered saline, the sections were incubated with biotinylated polyclonal goat anti-rabbit secondary antibody (\#A6154; 1:5,000 dilution; Sigma-Aldrich) followed by an avidin-biotin-peroxidase complex (Sigma-Aldrich) for a further $2 \mathrm{~h}$, prior to being visualized with diaminobenzidine. Finally, the sections were mounted on slides (in the dark for immunofluorescence) and analyzed under a microscope (IX83; Olympus Corporation, Beijing, China). Negative controls were set up by performing the experiments without the primary antibodies.

Evaluation of immunostaining. Image-Pro Plus 6.0 software (Media Cybernetics Inc., Silver Spring, MD, USA) was used to quantify the optical density value of OT in the PVN and SON and lamina I/II of the superficial dorsal horn of the lumbar enlargement. According to the rat brain stereotaxic atlas of Paxinos and Watson (12), the PVN and SON were located from 1.08 to $2.16 \mathrm{~mm}$ and from 0.92 to $1.44 \mathrm{~mm}$ posterior to the Bregma, respectively (15). The mean optical density of the OT staining was calculated by dividing the optical density with the measurement area.

Statistical analysis. Statistical analyses were performed using SPSS software, version 11.0 (SPSS Inc., Chicago, IL, USA). Comparisons of means between two groups were performed with Student's t-tests and among multiple groups with one-way analysis of variance followed by post hoc pairwise comparisons using Dunnett's tests. Data are presented as the mean \pm standard error of the mean. A two-tailed $\mathrm{P}<0.05$ was considered to indicate a statistically significant difference in this study.

\section{Results}

Hindpaw incision induces a reduction in OT levels in the $P V N$. To determine whether surgical incision affected OT in the PVN, OT in the PVN of animals with or without hindpaw incision was immunostained and the OT content was quantified using the optical density of the OT staining. As shown in Fig. 1, the OT content (optical density value) in the PVN at baseline (prior to incision) was $13.5 \pm 1.2(n=7)$. Following incision, the OT content in the PVN was $6.7 \pm 1.0,3.5 \pm 0.8$ and $4.8 \pm 0.9$ at $0.5,1.0$ and $3.0 \mathrm{~h}$, respectively $(\mathrm{n}=6$ at each time-point), indicating a significant decrease compared with the baseline $(\mathrm{P}<0.05)$. Between 6 and $24 \mathrm{~h}$ after incision, the OT content in the PVN returned to the baseline level $(n=6$ at each time-point) (Fig. 1). By contrast, the sham groups ( $n=5$ at each time-point) showed no significant differences in the OT 

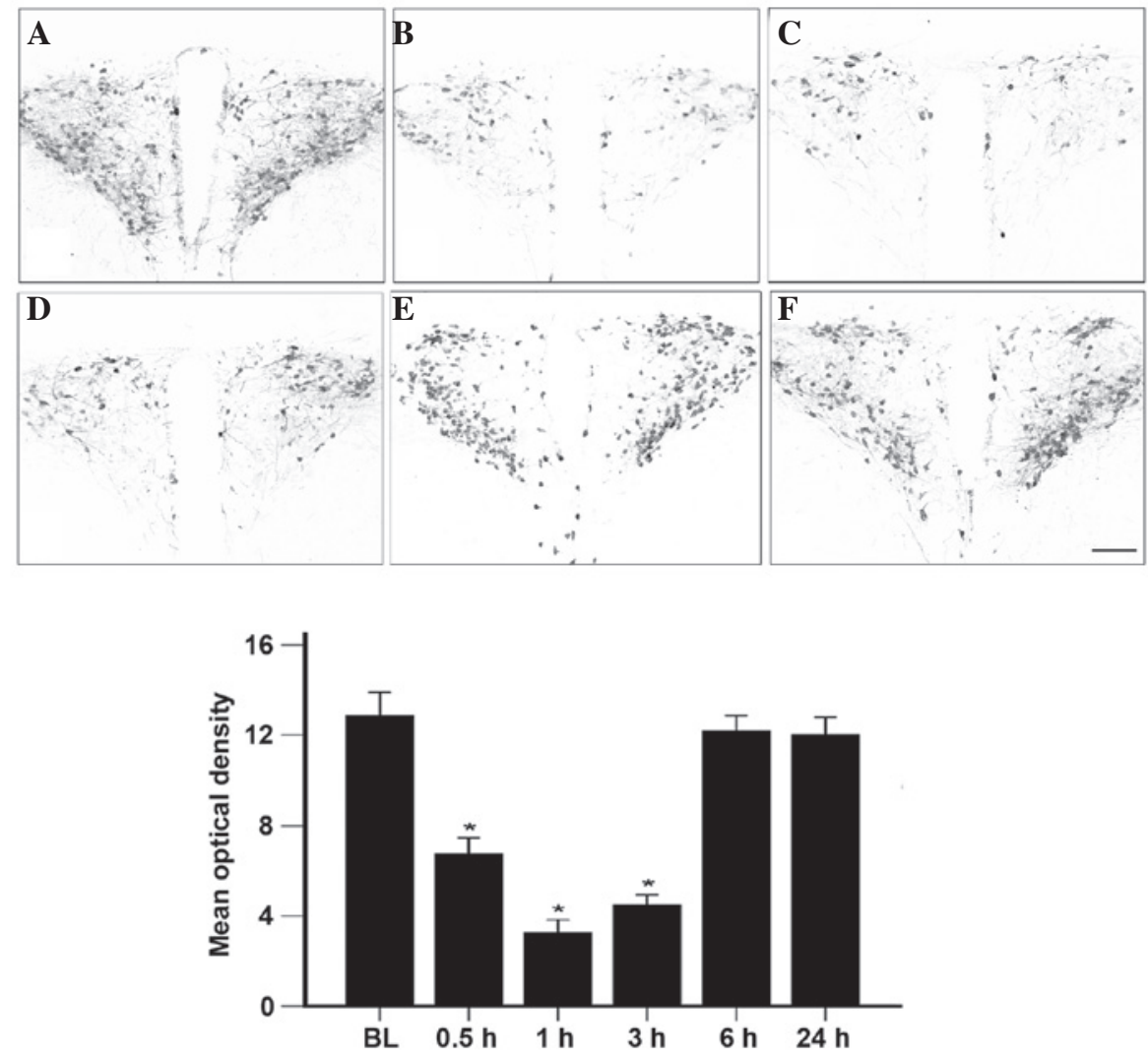

Figure 1. OT content in the PVN prior and subsequent to hindpaw incision. Immunohistochemical staining for OT was performed in the PVN in rats at (A) BL (prior to incision); and (B) $0.5 \mathrm{~h}$; (C) $1.0 \mathrm{~h}$; (D) $3.0 \mathrm{~h}$; (E) $6.0 \mathrm{~h}$ and (F) $24.0 \mathrm{~h}$ after incision. The OT content was quantified using the optical density of the OT staining, which is shown in the bar graph. Data are presented as the mean \pm standard error of the mean. Scale bar, $200 \mu \mathrm{m}$. Magnification, $\mathrm{x} 200$. ${ }^{*} \mathrm{P}<0.05 \mathrm{vs}$. BL. OT, oxytocin; PVN, paraventricular nucleus; BL, baseline.

$\mathbf{A}$
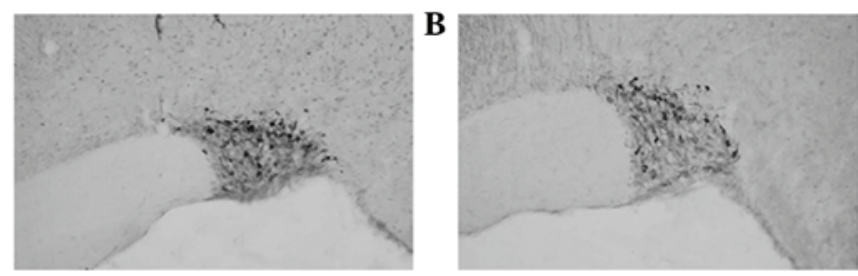

C

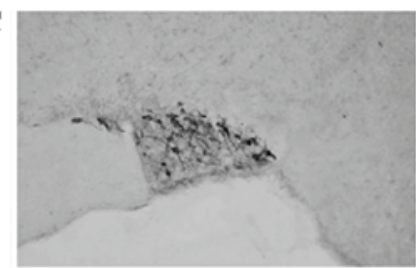

D

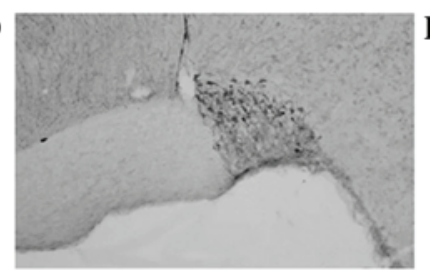

E

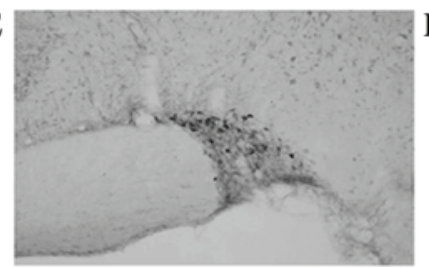

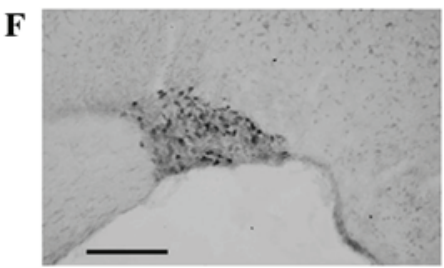

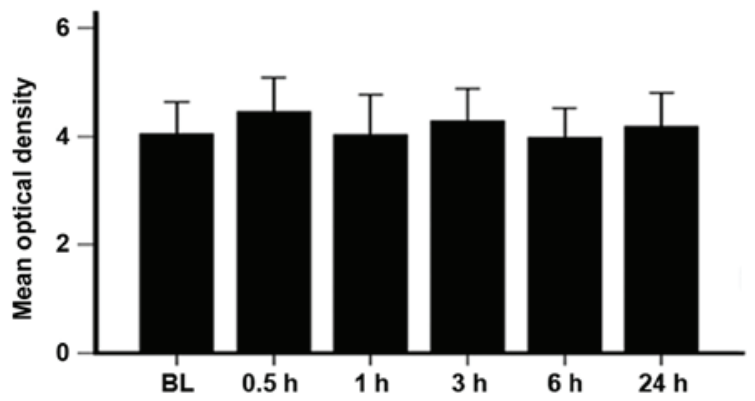

Figure 2. OT content in the SON prior and subsequent to hindpaw incision. Immunohistochemical staining for OT was performed in the SON in rats at (A) BL (prior to incision); and (B) $0.5 \mathrm{~h}$; (C) $1.0 \mathrm{~h}$; (D) $3.0 \mathrm{~h}$; (E) $6.0 \mathrm{~h}$ and (F) $24.0 \mathrm{~h}$ after incision. The OT content was quantified using the optical density of the OT staining, which is shown in the bar graph. Data are presented as the mean \pm standard error of the mean. Scale bar, $200 \mu \mathrm{m}$. Magnification, x200. BL, baseline, OT, oxytocin; SON, supraoptic nucleus. 

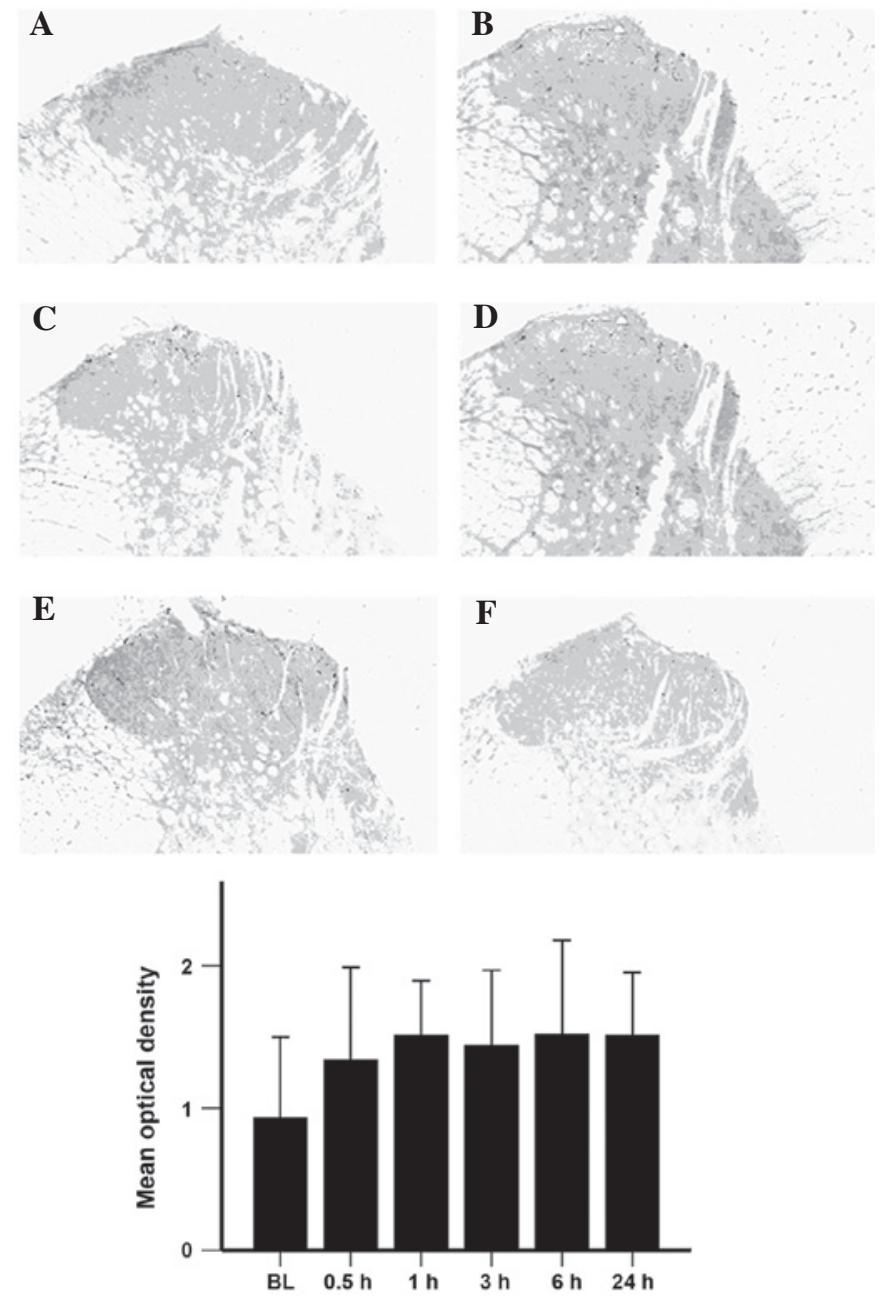

Figure 3. OT content in the spinal dorsal horn prior and subsequent to hindpaw incision. Immunohistochemical staining for OT was performed in the spinal dorsal horn in rats at (A) BL (prior to incision); and (B) $0.5 \mathrm{~h}$; (C) $1.0 \mathrm{~h}$; (D) $3.0 \mathrm{~h}$; (E) $6.0 \mathrm{~h}$ and (F) $24.0 \mathrm{~h}$ after incision. The OT content was quantified using the optical density of the OT staining, which is shown in the bar graph. Data are presented as the mean \pm standard error of the mean. Scale bar, $200 \mu \mathrm{m}$. Magnification, x200. BL, baseline; OT, oxytocin.

content in the PVN prior to and subsequent to incision (data not shown).

Expression of $O T$ in the SON and spinal cord remains unchanged following hindpaw incision. As shown in Fig. 2, there was no significant difference in the OT content in the SON prior to and subsequent to incision. Notably, the dorsal horn of the spinal cord showed no significant difference in the OT content prior to and following incision, despite being an important center of pain modulation (Fig. 3).

Intracerebroventricular but not intrathecal injection of OT attenuates mechanical hypersensitivity following hindpaw incision. To explore the role of OT in mechanical hypersensitivity induced by incision, intrathecal or intracerebroventricular injections of OT were performed immediately subsequent to hindpaw incision. A dose-dependent inhibition of mechanical hypersensitivity was detected $30 \mathrm{~min}$ after intracerebroventricular injection of OT (100, 400 or $600 \mathrm{ng})$ and lasted for $3.0 \mathrm{~h}$ ( $\mathrm{n}=5$ per group) (Fig. 4A). By contrast,
A

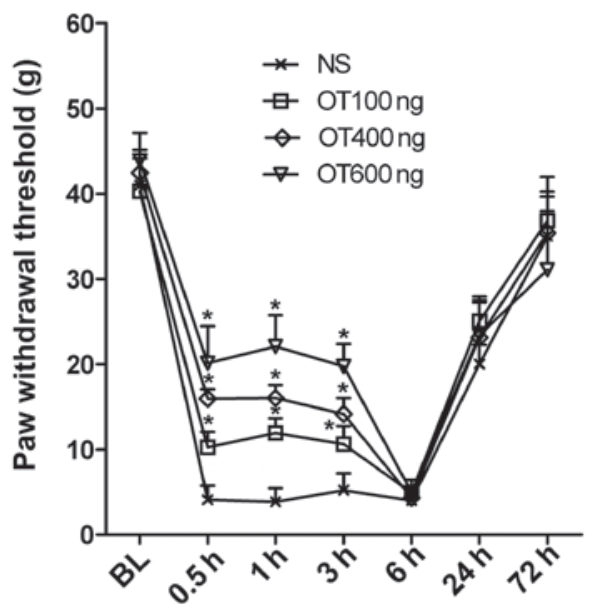

B

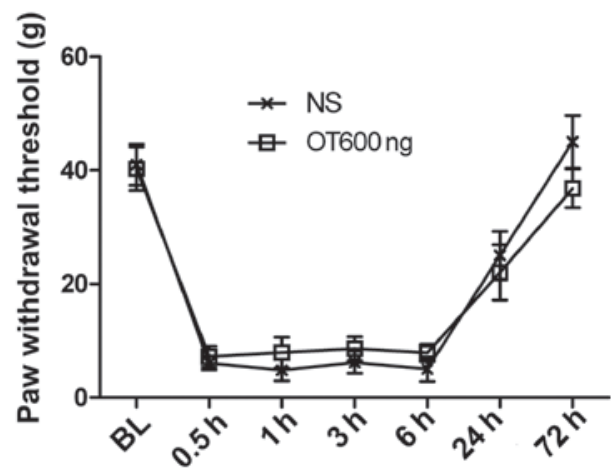

Figure 4. Effect of OT on incision-induced mechanical hypersensitivity of the hindpaw. The paw withdrawal threshold of the rats at BL (prior to incision) and $0.5,1.0,3.0,6.0,24.0$ and $72.0 \mathrm{~h}$ after incision was measured with (A) intracerebroventricular injection of NS or 100, 400 or $600 \mathrm{ng}$ OT or (B) intrathecal injection of NS or $600 \mathrm{ng}$ OT. ${ }^{*} \mathrm{P}<0.05$ vs. NS. OT, oxytocin; $\mathrm{BL}$, baseline; NS, normal saline.

no significant difference was noted between the intrathecal OT injection group (600 ng) and the control group ( $\mathrm{n}=5$ per group) (Fig. 4B).

\section{Discussion}

In the present study, it was shown that OT is involved in the response to incisional pain at the supraspinal level, but not at the spinal cord as expected. Following incision, a marked decrease in the OT content was observed in the PVN; however, no significant change in the OT content was noted at the spinal cord within $24.0 \mathrm{~h}$ of the incision. Administration of exogenous OT at the supraspinal level by intracerebroventricular injection attenuated mechanical hypersensitivity in a dose-dependent manner, while injection at the spinal level did not produce any analgesia. These results are noteworthy, given that the spinal cord is an important center of pain modulation.

OT can exert a wide spectrum of central and peripheral effects, including modulation of the neuroendocrine reflex and analgesia $(1,4)$. OT is synthesized in the PVN and SON and then transported to the peripheral circulation system or different regions of the central nervous system $(1,4)$. In the present study, it was found that the OT content in the PVN was significantly reduced between 0.5 and $3.0 \mathrm{~h}$ after inci- 
sion and returned to the baseline level after $6.0 \mathrm{~h}$. The OT content in the SON, however, was not altered significantly following incision. This is in agreement with the observation by Rousselot et al (16) that the OT that is involved in an anti-nociceptive or analgesic response is likely to stem from the PVN and not the SON.

OT fibers project to several regions involved in pain modulation in the nervous system $(17,18)$. It has been reported that $\geq 25 \%$ of OT-positive neurons in the PVN directly project to the superficial dorsal horn of the spinal cord (19). Furthermore, the spinal cord has overlap between OT fiber projections and the distribution of OT binding sites (6). Intrathecal injection of OT and electrical stimulation of the PVN can reduce the hypersensitivity of neuropathic or nociceptive responses in normal rats, and such effects can be inhibited by pretreatment with intrathecal injection of an OT antagonist (6). Condés-Lara et al $(20,21)$ suggested that intrathecal injection of OT could reduce the activation of primary A $\delta$ and $C$ fibers evoked by somatic stimulation or neuropathy. Yu et al (22) found that intrathecal administration of OT dose-dependently attenuated carrageenan-induced inflammatory pain. In the spinal cord, OT transported from the PVN activates $\gamma$-aminobutyric acid (GABA)-ergic interneurons, which in turn inhibit the activation of glutamatergic primary sensory neurons. Such presynaptic inhibition prevents the transmission of nociceptive signals to the brain (23-25). In addition to the increase in GABA release, changes in the reversal potential of GABAergic currents in nociceptive neurons contribute to the analgesic effects of OT (26-29). In combination, the previous studies indicate that OT contributes to pain relief at the spinal level; however, in the present study, it was found that the expression of OT in the ipsilateral lumbar enlargement of the spinal cord remained unchanged until $24 \mathrm{~h}$ after incision, and intrathecal injection of OT did not improve mechanical hypersensitivity. By contrast, intracerebroventricular injection of OT dose-dependently elevated the mechanical hypersensitivity threshold. As intrathecal injection acts at the spinal cord, the results from the present study suggest that OT attenuates acute incisional pain at the supraspinal level rather than at the spinal level (30). The PVN sends projections to numerous brain regions implicated in pain modulation, such as the PAG, raphe magnus and dorsal raphe nuclei (31-33). Injection of OT into the cerebral ventricle can elevate pain thresholds in rats and humans $(8,34)$. In addition, a previous study reported that OT could exert analgesic effects in neonatal rats during delivery, even when the descending OT fibers were cut by decerebration at the upper pons level (35). This suggests that OT in the brain can exert analgesic effects independently of the spinal cord and corroborates the findings from the present study.

The reduction of the OT content in the PVN following incision could be due to an alteration of OT transportation in the brain, as the OT content in the PVN was restored to the baseline level within $6 \mathrm{~h}$ of the incision. We aim to explore the underlying mechanisms in future studies.

In conclusion, the present study provides the first in vivo evidence, to the best of our knowledge, that OT in the PVN attenuates incision-induced mechanical allodynia at the supraspinal, but not the spinal, level. This suggests that OT is involved in supraspinal analgesia for postoperative pain.

\section{Acknowledgements}

This study was supported by a grant from the National Natural Science Foundation of China (no. 81070897). The authors would like to thank Ms. Dan Liu and Dr Jian-Wei Zhang for their technical support.

\section{References}

1. Gimpl G and Fahrenholz F: The oxytocin receptor system: structure, function, and regulation. Physiol Rev 81: 629-683, 2001.

2. Russell JA and Leng G: Sex, parturition and motherhood without oxytocin? J Endocrinol 157: 343-359, 1998.

3. Lee HJ, Macbeth AH, Pagani JH and Young WS III: Oxytocin: The great facilitator of life. Prog Neurobiol 88: 127-151, 2009. (Review.)

4. Lundeberg T, Uvnäs-Moberg K, Agren G and Bruzelius G: Anti-nociceptive effects of oxytocin in rats and mice. Neurosci Lett 170: 153-157, 1994.

5. Martínez-Lorenzana G, Espinosa-López L, Carranza M, Aramburo C, Paz-Tres C, Rojas-Piloni G and Condés-Lara M: PVN electrical stimulation prolongs withdrawal latencies and releases oxytocin in cerebrospinal fluid, plasma, and spinal cord tissue in intact and neuropathic rats. Pain 140: 265-273, 2008.

6. Miranda-Cardenas Y, Rojas-Piloni G, Martínez-Lorenzana G, Rodríguez-Jiménez J, López-Hidalgo M, Freund-Mercier MJ and Condés-Lara M: Oxytocin and electrical stimulation of the paraventricular hypothalamic nucleus produce antinociceptive effects that are reversed by an oxytocin antagonist. Pain 122: 182-189, 2006.

7. Padhy BM and Kumar VL: Inhibition of Calotropis procera latex-induced inflammatory hyperalgesia by oxytocin and melatonin. Mediators Inflamm 6: 360-365, 2005.

8. Madrazo I, Franco-Bourland RE, León-Meza VM and Mena I: Intraventricular somatostatin-14, arginine vasopressin, and oxytocin: analgesic effect in a patient with intractable cancer pain. Appl Neurophysiol 50: 427-431, 1987.

9. Yang J: Intrathecal administration of oxytocin induces analgesia in low back pain involving the endogenous opiate peptide system. Spine (Phila Pa 1976) 19: 867-871, 1994.

10. Zimmermann M: Ethical guidelines for investigations of experimental pain in conscious animals. Pain 16: 109-110, 1983.

11. Brennan TJ, Vandermeulen EP and Gebhart GF: Characterization of a rat model of incisional pain. Pain 64: 493-501, 1996.

12. Paxinos G and Watson C (eds): The Rat Brain in Stereotaxic Coordinates. 4th edition. Academic Press, Sydney, 1998.

13. Wang Y, Liu C, Guo QL, Yan JQ, Zhu XY, Huang CS and Zou WY: Intrathecal 5-azacytidine inhibits global DNA methylation and methyl-CpG-binding protein 2 expression and alleviates neuropathic pain in rats following chronic constriction injury. Brain Res 1418: 64-69, 2011.

14. Chaplan SR, Bach FW, Pogrel JW, Chung JM and Yaksh TL: Quantitative assessment of tactile allodynia in the rat paw. J Neurosci Methods 53: 55-63, 1994.

15. Zhao DQ and Ai HB: Oxytocin and vasopressin involved in restraint water-immersion stress mediated by oxytocin receptor and vasopressin $1 \mathrm{~b}$ receptor in rat brain. PLoS One 6: e23362, 2011.

16. Rousselot P, Papadopoulos G, Merighi A, Poulain DA and Theodosis DT: Oxytocinergic innervation of the rat spinal cord. An electron microscopic study. Brain Res 529: 178-184, 1990.

17. Hashimoto H, Fukui K, Noto T, Nakajima T and Kato N: Distribution of vasopressin and oxytocin in rat brain. Endocrinol Jpn 32: 89-97, 1985.

18. Sofroniew MV, Weindl A, Schrell U and Wetzstein R: Immunohistochemistry of vasopressin, oxy tocin and neurophysin in the hypothalamus and extrahypothalamic regions of the human and primate brain. Acta Histochem Supp 24: 79-95, 1981.

19. Sawchenko PE and Swanson LW: Immunohistochemical identification of neurons in the paraventricular nucleus of the hypothalamus that project to the medulla or to the spinal cord in the rat. J Comp Neurol 205: 260-272, 1982.

20. Condés-Lara M, González NM, Martínez-Lorenzana G, Delgado OL and Freund-Mercier MJ: Actions of oxytocin and interactions with glutamate on spontaneous and evoked dorsal spinal cord neuronal activities. Brain Res 976: 75-81, 2003. 
21. Condés-Lara M, Maie IA and Dickenson AH: Oxytocin actions on afferent evoked spinal cord neuronal activities in neuropathic but not in normal rats. Brain Res 1045: 124-133, 2005.

22. Yu SQ, Lundeberg T and Yu LC: Involvement of oxytocin in spinal antinociception in rats with inflammation. Brain Res 983: 13-22, 2003.

23. Robinson DA, Wei F, Wang GD, Li P, Kim SJ, Vogt SK, Muglia LJ and Zhuo M: Oxytocin mediates stress-induced analgesia in adult mice. J Physiol 540: 593-606, 2002.

24. Condés-Lara M, Rojas-Piloni G, Martínez-Lorenzana G Rodríguez-Jiménez J, López Hidalgo M and Freund-Mercier MJ: Paraventricular hypothalamic influences on spinal nociceptive processing. Brain Res 1081: 126-137, 2006.

25. Condés-Lara M, Rojas-Piloni G, Martínez-Lorenzana G, López-Hidalgo M and Rodríguez-Jiménez J: Hypothalamospinal oxytocinergic antinociception is mediated by GABAergic and opiate neurons that reduce A-delta and $\mathrm{C}$ fiber primary afferent excitation of spinal cord cells. Brain Res 1247: 38-49, 2009.

26. De Koninck Y: Altered chloride homeostasis in neurological disorders: a new target. Curr Opin Pharmacol 7: 93-99, 2007.

27. Price TJ, Cervero F, Gold MS, Hammond DL and Prescott SA Chloride regulation in the pain pathway. Brain Res Rev 60: 149-170, 2009.

28. Granados-Soto V, Arguelles CF and Alvarez-Leefmans FJ: Peripheral and central antinociceptive action of $\mathrm{Na}^{+}-\mathrm{K}^{+}-2 \mathrm{Cl}$ cotransporter blockers on formalin-induced nociception in rats. Pain 114: 231-238, 2005.
29. Valencia-de Ita S, Lawand NB, Lin Q, Castañeda-Hernandez G and Willis WD: Role of the $\mathrm{Na}^{+}-\mathrm{K}^{+}-2 \mathrm{Cl}^{-}$cotransporter in the development of capsaicin-induced neurogenic inflammation. J Neurophysiol 95: 3553-3561, 2006.

30. Yaksh TL and Rudy TA: Chronic catheterization of the spinal subarachnoid space. Physiol Behav 17: 1031-1036, 1976.

31. Antunes JL and Zimmerman EA: The hypothalamic magnocellular system of the rhesus monkey: an immunocytochemical study. J Comp Neurol 181: 539-565, 1978.

32. Buijs RM, De Vries GJ, Van Leeuwen FW and Swaab DF: Vasopressin and oxytocin: distribution and putative functions in the brain. Prog Brain Res 60: 115-122, 1983.

33. Jenkins JS, Ang VT, Hawthorn J, Rossor MN and Iversen LL: Vasopressin, oxytocin and neurophysins in the human brain and spinal cord. Brain Res 291: 111-117, 1984.

34. Yang J, Yang Y, Chen JM, Liu WY, Wang CH and Lin BC: Central oxytocin enhances antinociception in the rat. Peptides 28: 1113-1119, 2007.

35. Mazzuca M, Minlebaev M, Shakirzyanova A, Tyzio R, Taccola G, Janackova S, Gataullina S, Ben-Ari Y, Giniatullin R and Khazipov R: Newborn analgesia mediated by oxytocin during delivery. Front Cell Neurosci 5: 3, 2011. 